\title{
Low threshold Q-switched fiber laser incorporating titanium dioxide saturable absorber from waste material
}

\begin{abstract}
We experimentally demonstrate a passively Q-switched erbium-doped fiber laser incorporating microfiber-based titanium dioxide ( $\mathrm{TiO} 2)$ polymer composite saturable absorber. The anatase $\mathrm{TiO} 2$ is derived from natural ilmenite waste via alkaline fusion method. The $\mathrm{TiO} 2$ polymer composite is prepared using solvent exchange method and deposited onto tapered microfiber via dip coating technique. The passive Q-switching at $1558.3 \mathrm{~nm}$ with low threshold pump power of $16.4 \mathrm{~mW}$, average output power of $1.0 \mathrm{~mW}$ and pulse energy of $47.6 \mathrm{~nJ}$ are realized. The shortest pulse width of $7 \mu$ s with $22.56 \mathrm{kHz}$ repetition rate is recorded. This work opens up a new window for the generation of microsecond pulses using waste material.
\end{abstract}

Keyword: Titanium dioxide; Saturable absorber; Q-switched fiber laser; Ilmenite waste 